PROCEEDINGS OF THE

AMERICAN MATHEMATICAL SOCIETY

Volume 30, No. 1, September 1971

\title{
GENERALISATION OF THE MUIRHEAD-RADO INEQUALITY
}

\author{
D. E. DAYKIN
}

Abstract. For polynomials $f_{\beta}(x)$ of $n$ real variables $x$ $=\left(x_{1}, x_{2}, \cdots, x_{n}\right)$ of the form

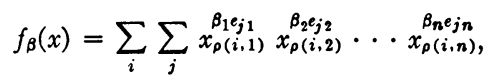

conditions are given which ensure that $f_{\alpha}(x) \leqq f_{\beta}(x)$ for all $x \geqq 0$.

Let $n$ be a positive integer and $N$ the set $\{1,2, \cdots, n\}$. Let $G$ be a group of permutations of $N$; so if there are $g$ permutations in $G$ we may denote them as $\left\{\left(\rho_{i 1}, \rho_{i 2}, \cdots, \rho_{i n}\right), 1 \leqq i \leqq g\right\}$. We write $\rho(i, k)$ or $\rho i k$ for the integer $\rho_{i k}$ of $N$. Let $V$ be an unordered set of $v$ ordered $n$ tuples $V=\left\{\left(e_{j 1}, e_{j 2}, \cdots, e_{j n}\right), 1 \leqq j \leqq v\right\}$ of real numbers $e_{j k}$. We say that $V$ is unaltered by $G$ if it is unaltered by permuting the elements of every one of its $n$-tuples by any permutation of $G$. If $\beta=\left(\beta_{1}, \beta_{2}, \cdots\right.$, $\left.\beta_{n}\right)$ is an ordered $n$-tuple of real numbers $\beta_{k}$ we write $H_{G}(\beta)$ for the convex hull of the set $\left\{\left(\beta_{\rho i 1}, \beta_{\rho i 2}, \cdots, \beta_{\rho i n}\right), 1 \leqq i \leqq g\right\}$. Finally if $x=\left(x_{1}, x_{2}, \cdots, x_{n}\right)$ is an ordered $n$-tuple of positive real variables $x_{k}>0$ we put

$$
f_{G, V, \beta}(x)=\sum_{1 \leqq i \leqq g} \sum_{1 \leqq j \leqq v} x_{\rho i 1}^{\beta_{1} e_{j 1}} x_{\rho i 2}^{\beta_{2} e_{j 2}} \cdots x_{\rho i n}^{\beta_{n} e_{j n}} .
$$

The object of this note is to prove the

THEOREM. If $V$ is unaltered by $G$ and if $\alpha \in H_{G}(\beta)$ then

$$
f_{G, V, \alpha}(x) \leqq f_{G, V, \beta}(x) \quad \text { for all } x>0 .
$$

Proof. That $\alpha \in H_{G}(\beta)$ means $[1, \S 6]$ that there exist real numbers $t_{h} \geqq 0$ with

$$
\sum_{1 \leqq h \leqq g} t_{h}=1
$$

such that

$$
\alpha_{k}=\sum_{1 \leqq h \leqq g} t_{h} \beta_{\rho h k} \quad \text { for } 1 \leqq k \leqq n .
$$

Received by the editors August 11, 1970.

A MS 1969 subject classifications. Primary 2652, 6070, 5230.

Key words and phrases. Convex hull, inequality, Muirhead, permutation group, polynomial, Rado, symmetric function. 
Thus

$$
\begin{aligned}
& f_{G, V, \alpha}(x)=\sum_{i} \sum_{j} \prod_{h}\left(x_{\rho i 1}^{\beta_{\rho h 1} e_{j 1}} \cdots x_{\rho i n}^{\beta_{\rho h n} e_{j n}}\right)^{t_{h}} \\
& \leqq \sum_{i} \sum_{j} \sum_{h} t_{h}\left(x_{\rho i 1}^{\beta_{\rho h 1} e_{j 1}} \cdots x_{\rho i n}^{\beta_{\rho h n} e_{j n}}\right) \\
& =\sum_{h} t_{h} \sum_{i} \sum_{j} x_{\rho(i, \rho}^{\left.\beta_{1} e_{j} \rho^{-1}(h, 1)\right)} \cdots x_{\rho(i, \rho}^{\beta_{n} e_{j}^{-1}(h, n)} \\
& =\sum_{h} t_{h} \sum_{i} \sum_{j} x_{\rho i 1}^{\beta_{1} e_{j \rho}^{-1}(h, 1)} \cdots x_{\rho i n}^{\beta_{n} e_{j \rho}{ }^{-1}(h, n)} \\
& =\sum_{h} t_{h} f_{G, V, \beta}(x) \\
& =f_{G, V, \beta}(x) \text {. }
\end{aligned}
$$

To get (5) we substitute (4) in $f_{G, V, \alpha}(x)$, which is similar to (1). Then (6) follows by multiple application of the generalised arithmetic-geometric mean inequality [2, Theorem 45]. We get equality in (2) only if we get equality in each of the applications of the inequality. To get (7) from (6) we permute the factors in the bracket by the inverse $\rho^{-1}$ of $\left(\rho_{h 1}, \cdots, \rho_{h n}\right)$. Since $G$ is a group, this inverse lies in $G$, and applying this inverse to every permutation of $G$ does not change $G$, hence we get (8). Because $V$ is unaltered by $G$, the summation over $j$ in (8) is simply summation over all the $n$-tuples of $V$, and so by inspection of (1) we get (9). Then (10) follows from (3), and the theorem is proved.

When $V$ is the set of all distinct permutations of $n$ given real numbers, it is clear that $V$ is unaltered by any $G$. This is true in particular when $1 \leqq m \leqq n$ and $V$ is the set of all $\left(\begin{array}{l}n \\ m\end{array}\right)$ permutations of the $n$-tuple $(1,1, \cdots, 1,0, \cdots, 0)$ with $m$ ones. Let $S_{m}\left(x_{1}, \cdots, x_{n}\right)$ denote the $m$ th elementary symmetric function on $x_{1}, \cdots, x_{n}$, so $S_{1}=x_{1}+x_{2}+\cdots+x_{n}$ and $S_{n}=x_{1} x_{2} \cdots x_{n}$; this case is the

Corollary. If $\alpha \in H_{G}(\beta)$ then

$$
\sum_{i} S_{m}\left(x_{\rho i 1}^{\alpha_{1}}, \cdots, x_{\rho i n}^{\alpha_{n}}\right) \leqq \sum_{i} S_{m}\left(x_{\rho i 1}^{\beta_{1}}, \cdots, x_{\rho i n}^{\beta_{n}}\right) \quad \text { for all } x>0 .
$$

The case $m=n$ of the corollary was proved by R. Rado [4], thus generalising the case $m=n$ and $G$ the group of all permutations which was proved by R. F. Muirhead [3] fifty years earlier. Both of them proved for their case that (11) implied $\alpha \in H_{G}(\beta)$, but this is not true in general. For example, if $G$ is the group of all permutations, and $V$ looks like the unit $n \times n$ matrix, the theorem tells that 


$$
\alpha \in H_{G}(\beta) \Rightarrow \sum_{k=1}^{n} y^{\alpha_{k}} \leqq \sum_{k=1}^{n} y^{\beta_{k}} \quad \text { for all } y>0 \text {. }
$$

However, with $n=4$ we have $(14,8,2,1) \notin H_{G}(15,6,4,0)$, as can be checked from [4], (3), even though

$$
\begin{array}{r}
y^{15}+y^{6}+y^{4}+y^{0}-\left(y^{14}+y^{8}+y^{2}+y^{1}\right) \\
=\frac{1}{4}(y-1)^{2}\left\{4 y^{13}+4 y^{12}+3 y^{11}+4 y^{10}+2 y^{9}+2 y^{8}+4 y^{7}+y\right. \\
\left.+y\left(y^{5}-1\right)^{2}+2(y+1)\left(y^{4}-1\right)^{2}+2\left(y^{3}-1\right)^{2}\right\} \geqq 0 \\
\text { for all } y>0 .
\end{array}
$$

Other inequalities of this nature are given in [5].

\section{REFERENCES}

1. T. Bonnesen and W. Fenchel, Theorie der konvexen Körper, Springer, Berlin, 1934.

2. G. H. Hardy, J. E. Littlewood and G. Pólya, Inequalities, Cambridge Univ. Press, New York, 1934.

3. R. F. Muirhead, Some methods applicable to identities and inequalities of symmetric algebraic functions of $n$ letters, Proc. Edinburgh Math. Soc. 21 (1903), 144-157.

4. R. Rado, An inequality, J. London Math. Soc. 27 (1952), 1-6. MR 13, 539.

5. D. E. Daykin, Inequalities for functions of a cyclic nature, J. London Math. Soc. (to appear).

University of Reading, Reading, Berkshire, England 